\title{
A new measurement of the neutron detection efficiency for the Nal Crystal Ball detector
}

(A2 Collaboration at MAMI)

M. Martemianov ${ }^{1}$, V. Kulikov ${ }^{1 *}$, B. T. Demissie ${ }^{2}$, Z. Marinides ${ }^{2}$, C. S. Akondi ${ }^{3}$, J. R. M. Annand ${ }^{4}$, H. J. Arends ${ }^{5}$, R. Beck ${ }^{6}$, N. Borisov ${ }^{7}$, A. Braghieri ${ }^{8}$, W. J. Briscoe ${ }^{2}$, S. Cherepnya ${ }^{9}$, C. Collicott ${ }^{5}, 10$, S. Costanza ${ }^{8,11}$, E. J. Downie ${ }^{2,5}$, M. Dieterle ${ }^{12}$, M. I. Ferretti Bondy ${ }^{5}$, L. V. Fil'kov' ${ }^{9}$, S. Garni ${ }^{12}$, D. I. Glazier', D. Glowa ${ }^{13}$, W. Gradl', G. Gurevich ${ }^{14}$, D. Hornidge ${ }^{15}$, G. M. Huber ${ }^{16}$, A. Kaeser ${ }^{12}$, V. L. Kashevarov ${ }^{5}$, I. Keshelashvili ${ }^{12}$, R. Kondratiev ${ }^{14}$, M. Korolija ${ }^{17}$, B. Krusche ${ }^{12}$, A. Lazarev ${ }^{7}$, J. M. Linturi ${ }^{5}$ V. Lisin ${ }^{14}$, K. Livingston ${ }^{4}$, I. J. D. MacGregor ${ }^{4}$, D. M. Manley ${ }^{3}$, P. P. Martel ${ }^{5,15}$, D. G. Middleton ${ }^{5,15}$, R. Miskimen ${ }^{18}$, A. Mushkarenkov ${ }^{18}$, A. Neganov ${ }^{7}$, A. Neiser ${ }^{5}$, M. Oberle ${ }^{12}$, M. Ostrick ${ }^{5}$, P. Ott ${ }^{5}$, P. B. Otte ${ }^{5}$, B. Oussena ${ }^{2,5}$, P. Pedroni $^{8}$, A. Polonski ${ }^{14}$, S. Prakhov ${ }^{5}$, G. Ron ${ }^{19}$, T. Rostomyan ${ }^{12}$, A. Sarty ${ }^{10}$, D. M. Schott ${ }^{2}$, S. Schumann ${ }^{5}$, V. Sokhoyan ${ }^{2,5}$, O. Steffen ${ }^{5}$, I. I. Strakovsky ${ }^{2}$, Th. Strub ${ }^{12}$, I. Supek ${ }^{17}$, M. Thiel ${ }^{5}$, A. Thomas ${ }^{5}$, M. Unverzagt ${ }^{5}$, Yu. A. Usov ${ }^{7}$, S. Wagner ${ }^{5}$, D. P. Watts ${ }^{13}$, J. Wettig ${ }^{5}$, D. Werthmüller ${ }^{12}$, L. Witthauer ${ }^{12}$ and M. Wolfes ${ }^{5}$ 
${ }^{1}$ Institute for Theoretical and Experimental Physics SRC "Kurchatov Institute", Moscow 117218, Russia

${ }^{2}$ The George Washington University, Washington, DC 20052-0001, USA

${ }^{4}$ SUPA School of Physics and Astronomy, University of Glasgow, Glasgow G12 8QQ, United Kingdom

${ }^{5}$ Institut für Kernphysik, University of Mainz, D-55099 Mainz, Germany

${ }^{6}$ Helmholtz-Institut für Strahlen- und Kernphysik, University of Bonn, D-53115 Bonn, Germany

${ }^{7}$ Joint Institute for Nuclear Research, 141990 Dubna, Russia

${ }^{8}$ INFN Sezione di Pavia, I-27100 Pavia, Italy

${ }^{11}$ Dipartimento di Fisica, Università di Pavia, I-27100 Pavia, Italy

${ }^{9}$ Lebedev Physical Institute, 119991 Moscow, Russia

${ }^{10}$ Department of Astronomy and Physics, Saint Mary's University, Halifax, Nova Scotia B3H 3C3, Canada

${ }^{12}$ Department für Physik, University of Basel, CH-4056 Basel, Switzerland

${ }^{13}$ SUPA School of Physics, University of Edinburgh, Edinburgh EH9 3JZ, United Kingdom

${ }^{14}$ Institute for Nuclear Research, 125047 Moscow, Russia

${ }^{15}$ Mount Allison University, Sackville, New Brunswick E4L 1E6, Canada

${ }^{16}$ University of Regina, Regina, Saskatchewan S4S OA2, Canada

${ }^{17}$ Rudjer Boskovic Institute, HR-10000 Zagreb, Croatia

${ }^{3}$ Kent State University, Kent, Ohio 44242-0001, USA

${ }^{18}$ University of Massachusetts, Amherst, Massachusetts 01003, USA

${ }^{19}$ Racah Institute of Physics, Hebrew University of Jerusalem, Jerusalem 91904, Israel

ABSTRACT: We report on a measurement of the neutron detection efficiency in NaI crystals in the Crystal Ball detector obtained from a study of single $\pi^{0}$ photoproduction on deuterium using the tagged photon beam at the Mainz Microtron. The results were obtained up to a neutron energy of $400 \mathrm{MeV}$. They are compared to previous measurements made more than 15 years ago at the pion beam at the BNL AGS.

KeYWORDS: Performance of High Energy Physics Detectors; Calorimeters; NaI Neutron Detector; Photoproduction.

\footnotetext{
${ }^{*}$ Corresponding author, E-mail: kulikov@itep.ru
} 


\section{Contents}

1. Introduction 1

2. Experimental setup 2

3. Overview of the method 3

4. Photon Tagger

5. Selection of the single neutral pion

6. CB neutron detection efficiency

7. Neutron interaction characteristics 9

8. Discussion and conclusion 9

9. Acknowledgements 10

\section{Introduction}

The A2 Collaboration at MAMI is engaged in a program [1] to study $\gamma$ n-interactions for which neutron detection is of crucial importance. The A2 experiment consists of several detector systems described below. Two of them, the Crystal Ball (CB) and TAPS, have a good neutron detection efficiency. The NaI CB detector was built originally at SLAC in the mid 1970s for use with colliding $\mathrm{e}^{+} \mathrm{e}^{-}$beams at SPEAR [2]. Later the CB was involved in experiments at DORIS and Brookhaven National Laboratory before arriving at MAMI where it demonstrates an excellent performance for photon and charged particle detection. Neutron detection with this detector has been studied less. The CB neutron detection efficiency was measured previously in a 1997-1998 run at BNL [3] using the reaction $\pi^{-} p \rightarrow \pi^{0} n$. There are very few references in the literature to neutron interactions in NaI. A short summary of this topic can be found in [丹]. Previous measurements are not directly applicable to the current status of the CB because of possible aging effects, differences in thresholds and in methods of analyzing the data. In this paper, we describe a new study of the CB neutron detection efficiency based on measurements of single $\pi^{0}$ photoproduction on a liquid deuterium target using the tagged photon beam at MAMI. 


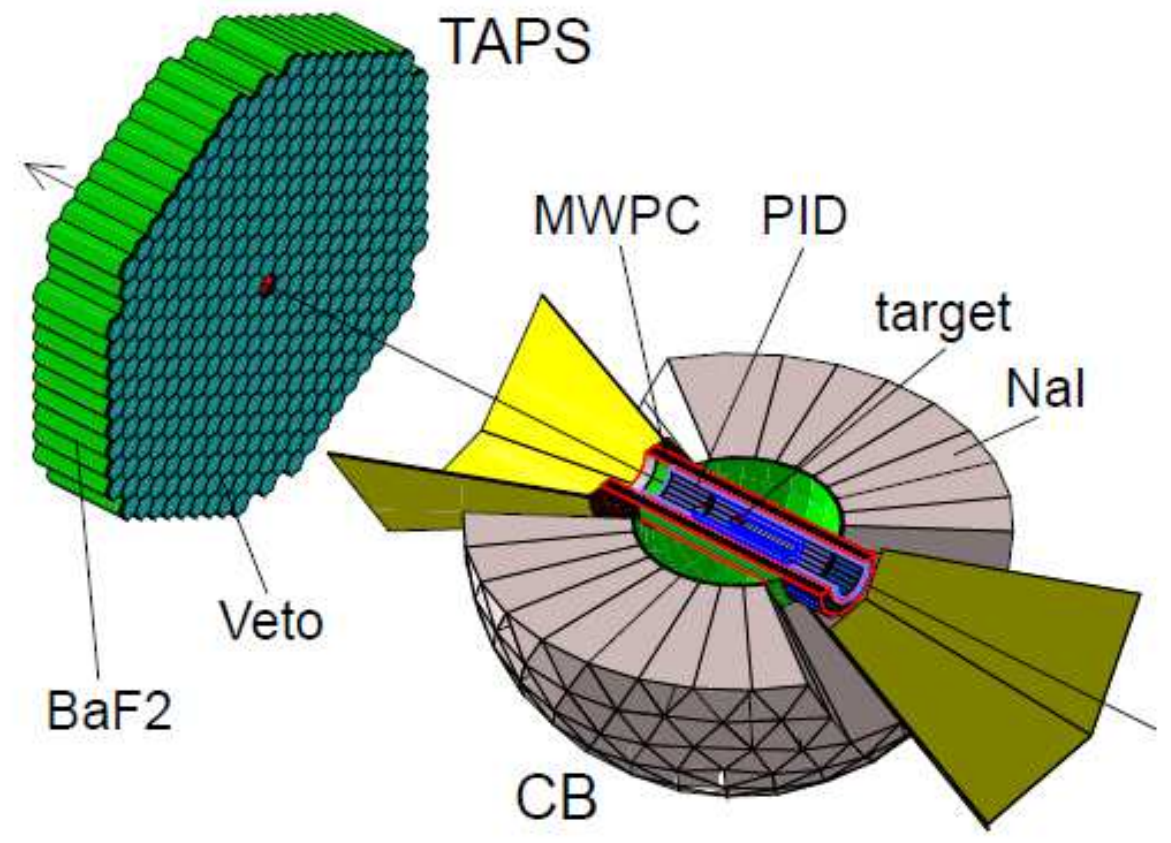

Figure 1. Layout of the A2 detector systems.

\section{Experimental setup}

The measurements were performed at the tagged photon facility of the Mainz Microtron accelerator (MAMI) [阿. An electron beam was used to produce bremsstrahlung photons, which were tagged with the upgraded Glasgow magnetic spectrometer [6]. The target was a Kapton cylinder of $4 \mathrm{~cm}$ diameter and $10 \mathrm{~cm}$ length filled with liquid deuterium. Photons, charged pions, and recoil nucleons, produced in the target, were detected with an almost $4 \pi$ electromagnetic calorimeter schematically shown in figure 1. It combined the CB and the forward TAPS detector [7], although the latter was not used in the current analysis.

The CB detector is a sphere consisting of 672 optically isolated $\mathrm{NaI}(\mathrm{Tl})$ crystals, shaped as truncated triangular pyramids, which point toward the center of the sphere. The crystals are arranged in two hemispheres that cover $93 \%$ of $4 \pi$ sr or polar angles of $20-160^{\circ}$, sitting outside a central spherical cavity with a radius of $25 \mathrm{~cm}$. This cavity holds the target and inner detectors. The NaI crystals have a length of $40.7 \mathrm{~cm}$, which is equal to 15.7 radiation lengths or $\sim 1$ hadron interaction length. The target was located in the center of the CB and was surrounded by a Particle Identification Detector (PID) [8] used to distinguish between neutral and different charged particles based on $d E / d x$ measurements. It was made of 24 scintillator bars $(50 \mathrm{~cm}$ long, $4 \mathrm{~mm}$ thick) arranged as a horizontal cylinder with a radius of $6 \mathrm{~cm}$. The PID was surrounded by two Cylindrical Multiwire Proportional Chambers (MWPC). The inner (outer) chamber has a radius of $7.4(9.45) \mathrm{cm}$ and a length of $57 \mathrm{~cm}$. Each chamber measured the three-dimensional coordinates of a charged-particle track as a result of a readout of three signals, one from a horizontal anode sense wire and the other two from spiral strip cathodes [9]. 


\section{Overview of the method}

For the neutron detection efficiency measurement, we chose the $\pi^{0}$ photoproduction on deuterium,

$$
\gamma+d \rightarrow \pi^{0}+p+n
$$

where the $\pi^{0}$ decays to two photons. The reaction kinematics is completely determined if the pion and proton are detected and the beam energy is known. The $\pi^{0}$ momentum is reconstructed using the energies and directions of the decay photons. The proton momentum is determined by its deposited energy in the CB and track direction in the MWPC and the photon beam energy is given by the tagging spectrometer. The reaction vertex was determined from the intersection of the proton track with the photon beam axis and allowed rejection of events from target walls. Then reaction (3.1) can be identified by selecting the neutron by missing mass. This procedure rejects events with more than one final-state pion and provides a reconstruction of the neutron momentum vector. If neutron momentum points to the $\mathrm{CB}$ (polar angles $30-150^{\circ}$ ), then a neutral hit in the $\mathrm{CB}$ in the same direction is searched for. Then the neutron detection efficiency is calculated as the ratio of registered neutral hits to all neutrons. The indicated angular range is chosen to be smaller than the full acceptance of the CB to eliminate edge regions that inevitably have efficiency losses due to uncertainties in predicted and real hit positions.

Apart from being kinematically completely determined, reaction (3.1) has other advantages. It has a large cross section and a broad energy and angle spectrum of neutrons. The energy spectrum extends from nearly zero, when the pion is produced on a proton with a spectator neutron, to the maximum energy from pion production on the neutron. The latter was limited by the beam energy and the available statistics. Reaction (3.1) was identified from four clusters in the CB and one hit in the PID. Two clusters arise from $\pi^{0}$ decay photons, the third one from proton energy loss, and the fourth from the neutron interaction with NaI. A cluster is defined as a group of not more than 13 adjacent $\mathrm{NaI}$ crystals each with deposited energy larger than $2 \mathrm{MeV}$ centered around the crystal with maximum energy deposition. The minimum cluster summed energy was set to $15 \mathrm{MeV}$ in the analysis. In this measurement, we selected events with three or four clusters in the CB and only one hit in PID to reduce background from multiple pion production. In the following sections, we describe all above mentioned steps in more detail.

\section{Photon Tagger}

For the neutron detection efficiency measurement, we used data from the deuterium run taken at MAMI [5] in March 2013. Bremsstrahlung photons, produced by the $883 \mathrm{MeV}$ electrons in a 10 $\mu \mathrm{m} \mathrm{Cu}$ foil and collimated by a $\mathrm{Pb}$ collimator $3 \mathrm{~mm}$ in diameter, were incident on a $10 \mathrm{~cm}$ long liquid deuterium target located at the center of the CB. The beam was $1 \mathrm{~cm}$ in diameter at the target. The energies of the incident photons were measured by detecting the post-bremsstrahlung electrons in the 352 channel focal-plane detector of the Glasgow-Mainz tagger [6]. The energy resolution of the tagged photons is determined mostly by the width of the tagger focal-plane detectors and by the electron-beam energy. For the present beam energies, the typical width of a tagger channel was about $2 \mathrm{MeV}$. The data were taken with a trigger that required the total energy deposit in the $\mathrm{CB}$ to exceed $40 \mathrm{MeV}$. Figure 2 shows the time distribution of tagged photons relative to the trigger. 


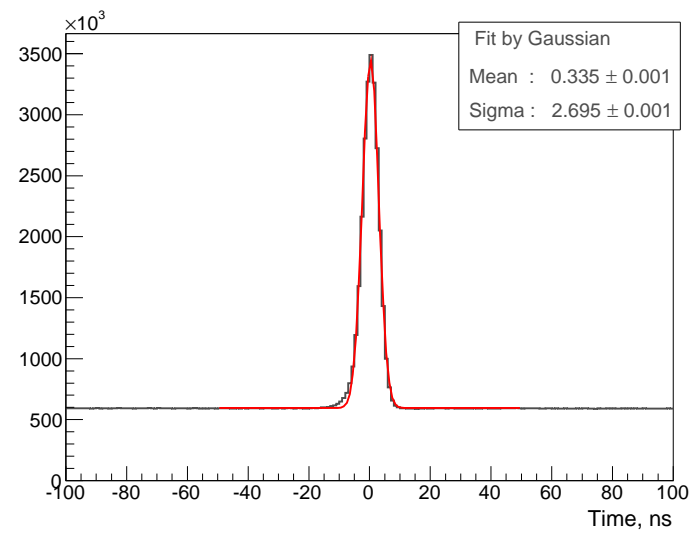

Figure 2. Time distribution of tagged photons.

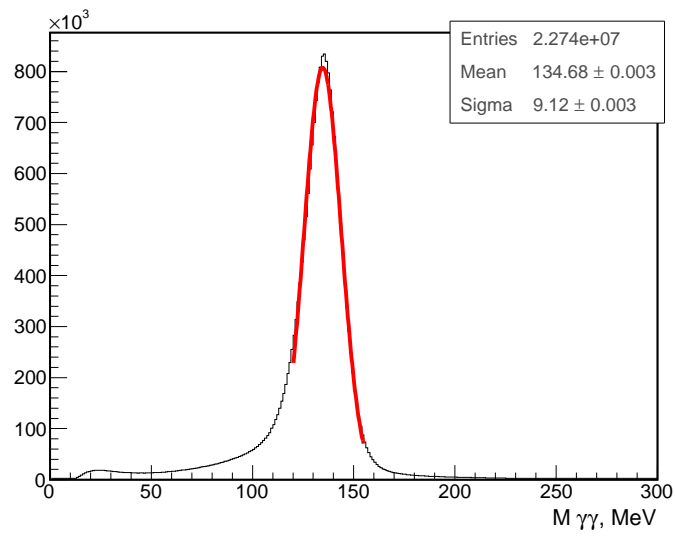

Figure 3. Invariant $\gamma \gamma$-mass. A Gaussian fit to the peak of the distribution is shown in red.

The peak near $0 \mathrm{~ns}$ is due to the prompt photons that produced the triggers. The entries outside and under this peak are due to random photons. The r.m.s. width of this peak is equal to $2.7 \mathrm{~ns}$ and is determined mostly by the intrinsic time resolution of the $\mathrm{CB}$ and time alignment of different channels. More accurate adjustment of the channel time offsets can improve the time resolution by a factor of two but it was not important for this analysis. We selected the prompt photons in a time window ( $-10 \mathrm{~ns},+10 \mathrm{~ns}$ ) and subtracted background produced by random photons for all distributions where the photon energy was used. The wide time range accepted by the tagger made it possible to use wide time windows for random photons (-230 ns to $-30 \mathrm{~ns}$ and $30 \mathrm{~ns}$ to $230 \mathrm{~ns}$ ) that resulted in a negligible subtraction error.

\section{Selection of the single neutral pion}

The first step for reconstruction of reaction (3.1) was neutral pion selection. This was done by choosing the photon pair, measured in the CB, with an invariant mass $m_{\gamma \gamma}$ closest to the $\pi^{0}$ mass $m_{\pi^{0}}$. An additional cut on the sum of energies of the two photons $E_{\gamma \gamma}>m_{\pi^{0}}$ rejects low-energy photons. The $m_{\gamma \gamma}$ distribution obtained with this cut is shown in figure 3 with a gaussian fit to the peak. The distribution demonstrates the excellent resolution of the NaI CB detector in $m_{\pi^{0}}$-mass of $9 \mathrm{MeV}$ (r.m.s.). For $\pi^{0}$ selection, we used a $120<m_{\gamma \gamma}<150 \mathrm{MeV}$ cut. Identification of the proton and measurement of its energy were done in a few steps. The proton track was required to have hits in the PID and the CB and space points in two MWPC compatible with a straight line that has a point of closest approach less than $10 \mathrm{~mm}$ from the beam axis within the volume of the target. The separation of protons from pions and electrons/positrons is displayed in figure 7 where the CB cluster energy is plotted along the horizontal axis and $d E / d x$ is plotted along the vertical axis. $d E / d x=E_{\mathrm{PID}} \sin \theta$, where $E_{\mathrm{PID}}$ is the energy deposited in the PID and $\theta$ is the track angle with respect to the beam axis. The factor $\sin \theta$ corrects the deposited energy for track length in the PID scintillator segments which are parallel to the beam axis. Ionization losses for protons depend on their energies. In figure $\sharp$, protons lie above the empirically drawn line while pions and electrons/positrons lie below. 


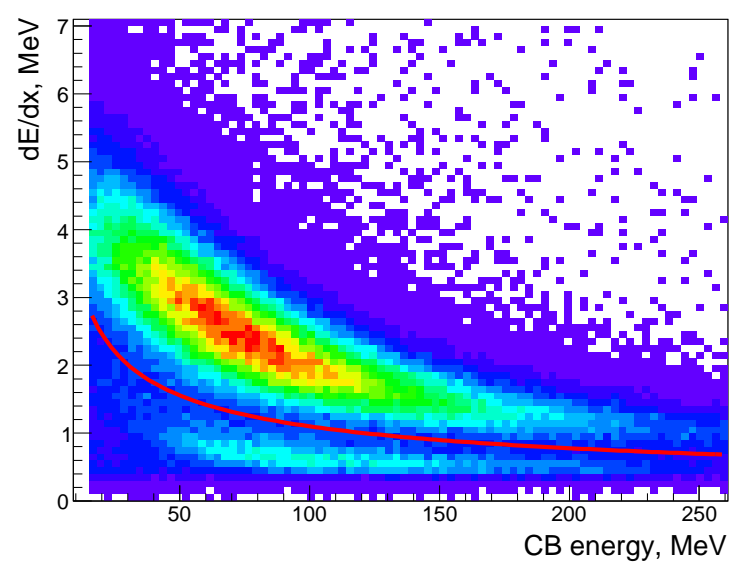

Figure 4. Pion/proton separation on the two dimensional plot $d E / d x$ as measured by PID vs. CB total energy. Protons lie above the empirically drawn line, pions - below.

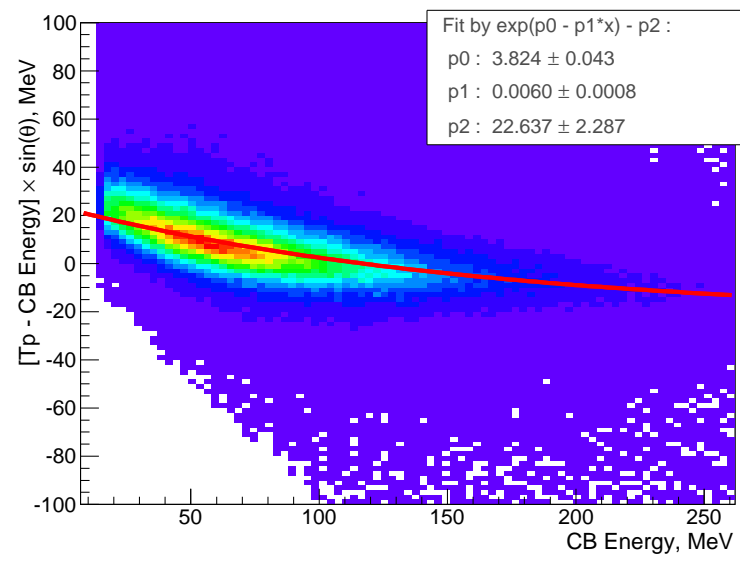

Figure 5. Proton energy correction for ionization losses and $\mathrm{NaI}$ light yield. $T_{\mathrm{p}}$ - proton kinetic energy at interaction point, $E$ - energy measured in the CB detector, $\theta$ - polar angle of proton track. Line - fitted correction curve.

Two effects must be taken into account to determine the proton energy at the interaction point. First, due to higher $d E / d x$ for protons that are stopped in the NaI crystals of the CB detector, the energy calibration for protons is different from that of minimum-ionizing electromagnetic showers. Second, the measured proton energy in the $\mathrm{CB}$ has to be corrected for ionization losses in detector materials between the interaction point and the CB crystals. These materials are $2 \mathrm{~cm}$ of liquid deuterium, $1 \mathrm{~mm} \mathrm{CH}$ of the target walls, $4 \mathrm{~mm}$ PID scintillator, and $1.5 \mathrm{~mm}$ stainless steel of the $\mathrm{CB}$ spherical inner wall. The proton energy at the interaction point was found by using the correction curve shown in figure 5, where $\left(T_{\mathrm{p}}-E\right) \sin \theta$ is plotted vs. the energy $(E)$ measured in the CB. Here $T_{\mathrm{p}}$ is the proton kinetic energy at the interaction point calculated from energy and momentum conservation for reaction (3.1) using photon and pion momentum vectors and the direction of the proton track. At each bin of CB energy, $\left(T_{\mathrm{p}}-E\right) \sin \theta$ was fitted with a gaussian and the maximum was found. Then these maxima at different energies were fitted with the function $\left(\exp \left(c_{1}-c_{2} E\right)-c_{3}\right)$ with three free parameters $c_{i}, i=1-3$. The efficient performance of this procedure is demonstrated in figure 6 , where distributions of the events over missing mass $M_{\text {miss }}$ in reaction (3.1) are given for a few intervals of the beam energy. Missing mass is defined as $M_{\text {miss }}=\sqrt{\left(P_{\gamma}+P_{\mathrm{d}}-P_{\pi^{0}}-P_{\mathrm{p}}\right)^{2}}$, where $P_{i}\left(i=\gamma, d, \pi^{0}, p\right)$ are the four-momentum vectors for the incident photon, target deuteron, neutral pion, and proton, respectively. Good agreement between the peak positions and the neutron mass is clearly seen in all four photon energy ranges.

\section{CB neutron detection efficiency}

Neutrons were selected by a cut on the missing mass $M_{\text {miss }}$ centered at the mass of a neutron $890<M_{\text {miss }}<990 \mathrm{MeV}$. The momentum vector for a selected neutron was calculated using energy and momentum conservation. This vector must point to a $\mathrm{CB}$ region slightly smaller than instrumented with $\mathrm{NaI}$ crystals to eliminate edge effects; that is, the angle $\theta$ of this vector with respect 

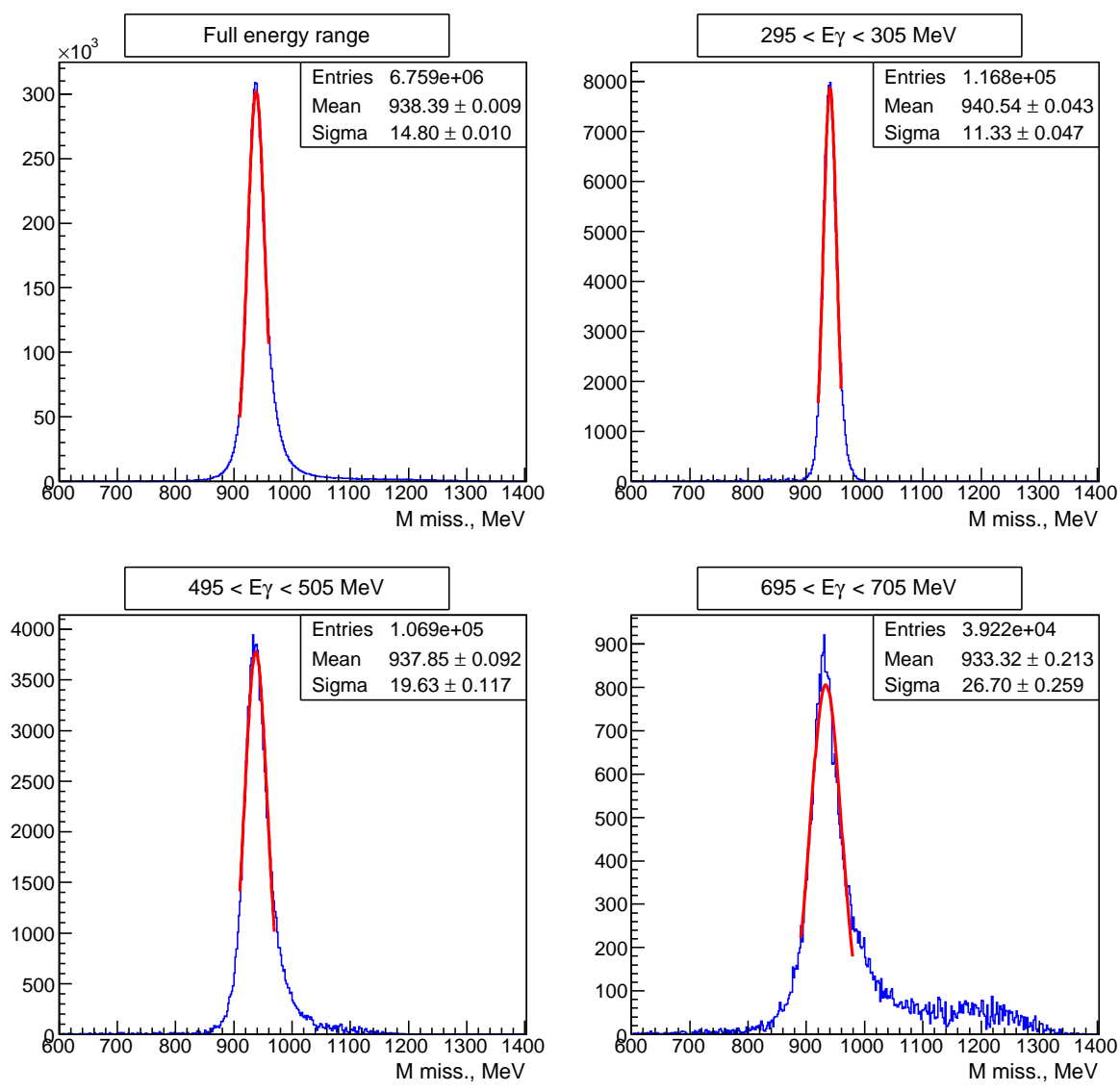

Figure 6. Missing mass distributions for the reaction $d\left(\gamma, \pi^{0} p\right) X$ for different incident photon energies. The peak position demonstrates good agreement with the neutron mass at all photon energies.

to the beam direction must be $30^{\circ}<\theta<150^{\circ}$. The angles $\lambda$ between this vector and the direction vectors for neutral hits in the $\mathrm{CB}$ were determined. Hits from protons and photons from $\pi^{0}$ decay were excluded from this procedure. The distribution over $\cos \lambda$ is given in figure 7 . It has a large peak at $\lambda=0$ signifying the real neutron hit in the NaI crystals and a small background of random hits. We chose $\cos \lambda=0.85$ as the boundary between the region of real neutron hits in the CB and background. The small uncertainty connected with this cut will be discussed later.

The energy distribution of neutrons from reaction (3.1) identified by missing mass and incident on the $\mathrm{CB}$ is shown by the solid line in figure 8. Those neutrons that gave hits in the $\mathrm{CB}$ with $\cos \lambda$ $<0.85$ have the energy distribution shown by the dashed line. The first distribution is peaked at low energy, which demonstrates the large contribution of spectator neutrons from pion photoproduction on the proton. The second distribution goes to zero at low energies because of the low detection efficiency for neutrons. The ratio of these two distributions gives the $\mathrm{CB}$ detection efficiency for neutrons shown in figure 9 and listed in Table 1 for 15 and $20 \mathrm{MeV}$ cluster energy thresholds. The detection efficiency is averaged over $\pm 10 \mathrm{MeV}$ relative to the listed energy values. The statistical and the two main systematic uncertainties for $15 \mathrm{MeV}$ threshold are also given. The statistical uncertainties vary from less than $0.1 \%$ at the lowest energy to $3 \%$ at $400 \mathrm{MeV}$. The increase results from the decrease in cross section for reaction (3.1) as proton and neutron energy increases. The 


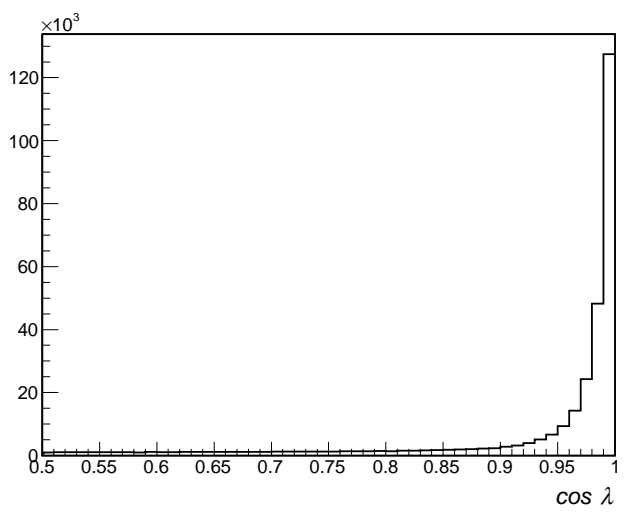

Figure 7. Cosine of the angle between the predicted neutron momentum vector and the direction vector for third neutral hit in $\mathrm{CB}$.

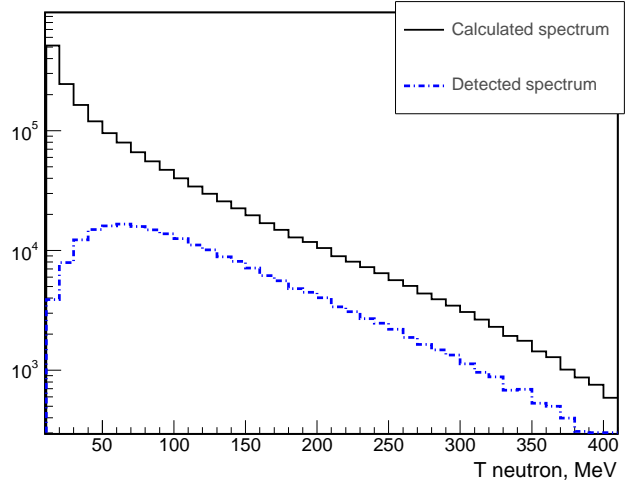

Figure 8. Kinetic energy for neutrons identified by missing mass - solid histogram. When a neutron hit is registered the dashed histogram is obtained.

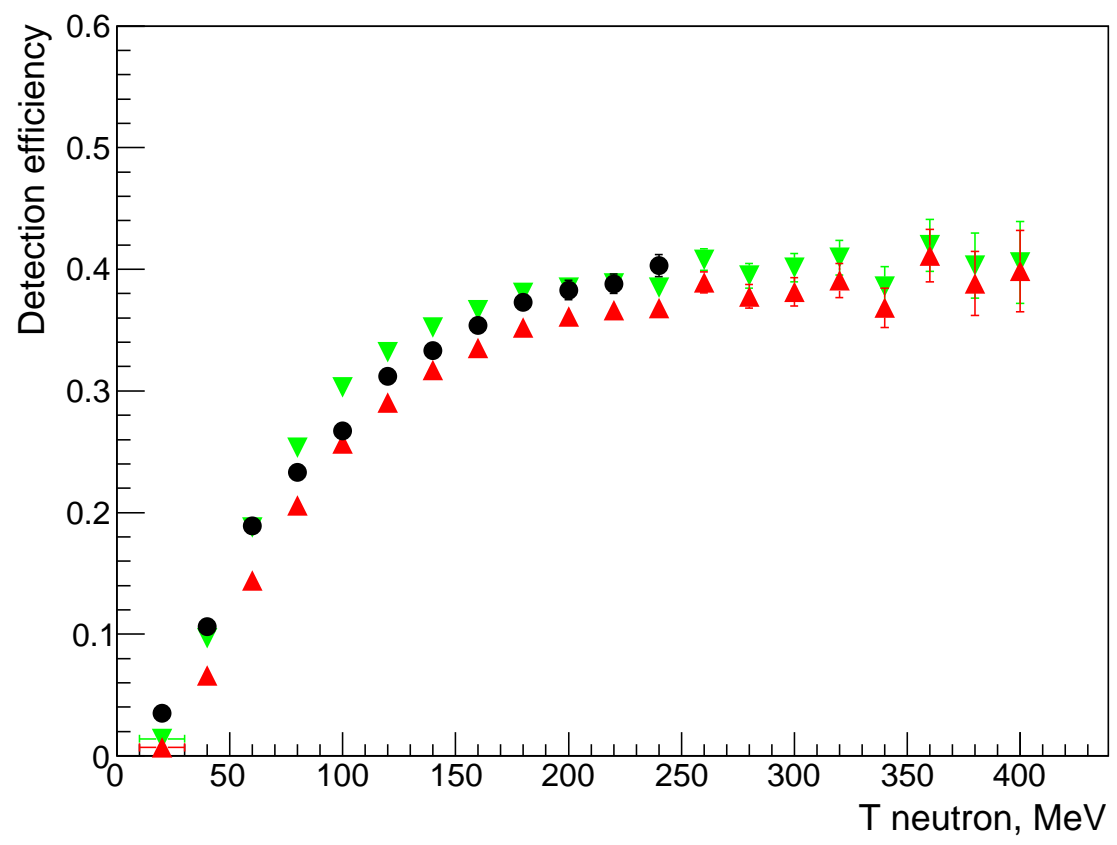

Figure 9. CB detection efficiency for neutrons as a function of neutron kinetic energy. Downward (upward) triangles - present measurements for a 15(20) MeV cluster energy threshold; circles - previous measurement at BNL in 1997-98 for a $20 \mathrm{MeV}$ threshold [3].

systematic uncertainty I was estimated by changing the cut on missing mass from (890 - 990) $\mathrm{MeV}$ to $(890$ - 1010) $\mathrm{MeV}$. The wider window gives slightly lower detection efficiency due to a small contamination from two-pion production. The smaller value of the lower boundary of the cut has negligible effect on the neutron detection efficiency. The systematic uncertainty II was estimated by changing the cut on $\cos \lambda$ from the standard one $\cos \lambda>0.85$ to $\cos \lambda>0.65$. Changing this 
Table 1. Neutron detection efficiency as a function of neutron kinetic energy. Column 2 gives the efficiency and statistical uncertainty for a $20 \mathrm{MeV}$ threshold, column 3 gives the efficiency and statistical uncertainty for a $15 \mathrm{MeV}$ threshold, columns 4 and 5 give systematic uncertainties I and II, and column 6 gives the total systematic uncertainty. See text for details.

\begin{tabular}{|c|c|c|c|c|c|}
\hline $\begin{array}{c}\text { Energy, } \\
\mathrm{MeV}\end{array}$ & $\begin{array}{c}\text { Efficiency, \% } \\
(20 \mathrm{MeV} \text { threshold })\end{array}$ & $\begin{array}{c}\text { Efficiency, \% } \\
(15 \mathrm{MeV} \text { threshold })\end{array}$ & $\begin{array}{c}\text { Sys. } \\
\text { I, \% }\end{array}$ & $\begin{array}{c}\text { Sys. } \\
\text { II, \% }\end{array}$ & $\begin{array}{c}\text { Sys. } \\
\text { total, \% }\end{array}$ \\
\hline 20 & $0.69 \pm 0.01$ & $1.39 \pm 0.01$ & 0.05 & 0.02 & 0.05 \\
40 & $6.6 \pm 0.1$ & $9.7 \pm 0.1$ & 0.1 & 0.1 & 0.2 \\
60 & $14.4 \pm 0.1$ & $18.8 \pm 0.1$ & 0.1 & 0.2 & 0.2 \\
80 & $20.6 \pm 0.2$ & $25.3 \pm 0.2$ & 0.1 & 0.3 & 0.3 \\
100 & $25.7 \pm 0.2$ & $30.3 \pm 0.2$ & 0.1 & 0.3 & 0.3 \\
120 & $29.0 \pm 0.3$ & $33.1 \pm 0.3$ & 0.2 & 0.3 & 0.3 \\
140 & $31.7 \pm 0.3$ & $35.2 \pm 0.3$ & 0.4 & 0.4 & 0.5 \\
160 & $33.5 \pm 0.4$ & $36.6 \pm 0.4$ & 0.4 & 0.4 & 0.6 \\
180 & $35.2 \pm 0.5$ & $38.1 \pm 0.5$ & 0.5 & 0.4 & 0.7 \\
200 & $36.1 \pm 0.5$ & $38.5 \pm 0.5$ & 0.5 & 0.5 & 0.7 \\
220 & $36.6 \pm 0.6$ & $38.8 \pm 0.6$ & 0.7 & 0.4 & 0.8 \\
240 & $36.8 \pm 0.7$ & $38.5 \pm 0.7$ & 0.6 & 0.4 & 0.7 \\
260 & $38.9 \pm 0.9$ & $40.8 \pm 0.9$ & 0.4 & 0.4 & 0.6 \\
280 & $37.8 \pm 1.0$ & $39.5 \pm 1.0$ & 0.7 & 0.7 & 1.0 \\
300 & $38.1 \pm 1.1$ & $40.1 \pm 1.1$ & 0.8 & 0.5 & 1.0 \\
320 & $39.1 \pm 1.4$ & $41.0 \pm 1.2$ & 0.6 & 0.7 & 1.0 \\
340 & $36.8 \pm 1.7$ & $38.6 \pm 1.7$ & 0.8 & 0.6 & 1.0 \\
360 & $41.1 \pm 2.1$ & $42.0 \pm 2.1$ & 0.5 & 0.4 & 0.6 \\
380 & $38.8 \pm 2.6$ & $40.3 \pm 2.6$ & 0.7 & 0.9 & 1.2 \\
400 & $39.8 \pm 3.4$ & $40.6 \pm 3.4$ & 0.4 & 0.3 & 0.7 \\
\hline
\end{tabular}

cut resulted in a higher detection efficiency as expected from the larger acceptance for neutron hits in the CB. Other cuts were also tested: $\pi^{0}$ - mass, window for prompt photons, position of interaction point in the liquid deuterium target, exclusion of dead channels (1\% of all channels). Each of these cuts had an order-of-magnitude smaller effect on neutron detection efficiency than I and II, mentioned above. The total systematic uncertainty was obtained as the quadrature sum of systematic uncertainties I and II and it is given in the last column of Table 1. For the most part, its value is close to the statistical uncertainty. Table 1 also demonstrates the sensitivity of neutron detection efficiency to the cluster energy threshold. An increase of the threshold from 15 to $20 \mathrm{MeV}$ results in a relative decrease of neutron detection efficiency by $30 \%$ at $40 \mathrm{MeV}$ neutron energy and by less than $5 \%$ for the energies higher than $300 \mathrm{MeV}$. In figure 9, the $\mathrm{CB}$ neutron detection efficiency measured more than 15 years ago [3] when the detector was in operation at BNL is also shown. The shape of its energy dependence for a $20 \mathrm{MeV}$ threshold is in reasonable agreement with the present measurement for a $15 \mathrm{MeV}$ threshold. Absolute values of CB neutron detection efficiency for the present measurement with a $20 \mathrm{MeV}$ threshold are $2-4 \%$ smaller than measured at BNL. 


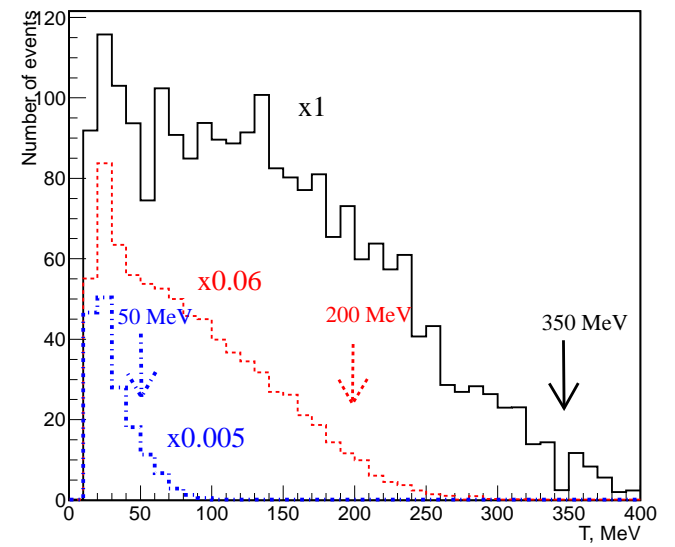

Figure 10. Energy deposited in the CB for three incident neutron energies (marked by arrows): $350 \pm$ $30 \mathrm{MeV}$ - solid black line, $200 \pm 20 \mathrm{MeV}$ - dashed red line, $50 \pm 10 \mathrm{MeV}$ - dash-dot blue line. The histograms were multiplied by the given factors to compensate for the strong energy dependence of the neutron flux.

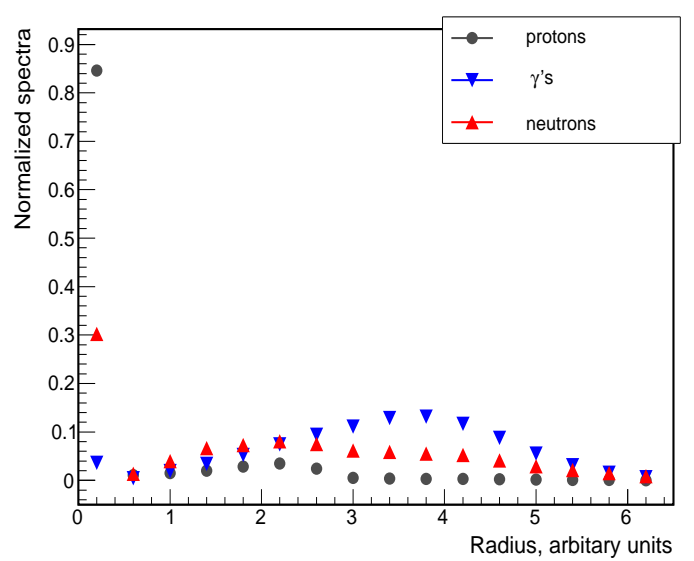

Figure 11. Energy weighted "radius" of the cluster for proton (black points), neutron (red upward triangles) and photon (blue downward triangles) induced showers. The peak at zero is due to clusters that consist of only one crystal.

\section{Neutron interaction characteristics}

Neutrons interacting in the $\mathrm{CB}$, which is not designed to be a totally absorbing hadronic calorimeter, generally deposit only part of their kinetic energy. This fact is illustrated by figure 10 where distributions over cluster energies recorded in the $\mathrm{CB}$ for hits identified as neutrons are given for three selected intervals of incident neutron energy: $50 \pm 10 \mathrm{MeV}, 200 \pm 20 \mathrm{MeV}$, and $350 \pm$ $30 \mathrm{MeV}$. For each incident energy, the response in the CB is distributed from threshold to a maximal energy that slightly exceeds the incident energy. This effect can be attributed to energy resolution and to some difference in calibration between hadron-induced and photon-induced showers. The latter was used to determine the neutron response. Figure 10 shows that the energy in a cluster induced by a neutron has limited utility for an estimation of the neutron energy; however, the shape of a cluster can be more informative. In figure 11, normalized distributions over cluster "radius" are given. The cluster "radius" is an energy-weighted sum of distances between the crystal with maximum energy and each remaining crystal in a cluster. The cluster "radius" is equal to zero for a cluster composed of only one crystal. For a cluster induced by a neutron the probability to have zero "radius" is nearly $30 \%$. It is much higher than for a photon cluster, for which the probability is only $4 \%$. This difference can be used to discriminate between neutron- and photon-induced showers. Proton-induced showers have an even larger probability for zero "radius" than neutroninduced showers. This information can also be used to control a sample of protons.

\section{Discussion and conclusion}

We have measured the detection efficiency in the NaI CB detector for neutrons in the kinetic en- 
ergy range 20 - $400 \mathrm{MeV}$ using $\pi^{0}$ photoproduction on deuterium. A previous measurement using the same detector was performed at BNL [3] in 1997-1998 with the $\pi^{-} p \rightarrow \pi^{0} n$ reaction. The present measurements agree closely with the previous measurements for the shape of the energy dependence of neutron detection efficiency but we find efficiencies that are 2-4\% smaller. This effect cannot be unambiguously explained as a result of possible degradation of the light yield of $\mathrm{NaI}$ crystals, which was part of the motivation for this study. Many hardware and software changes have occurred between these two measurements. Different energy calibration and clustering algorithms can also result in a slightly different neutron detection efficiency. The present measurement gives the up-to-date status of neutron detection with the $\mathrm{CB}$ detector and can be used in various physics analyses that involve neutrons and for improving the description of neutron detection in Monte Carlo simulations.

\section{Acknowledgements}

The authors wish to acknowledge the outstanding support of the accelerator group and operators of MAMI. This work was supported by the Deutsche Forschungsgemeinschaft (SFB 443, SFB 1044), the INFN-Italy, the Schweizerischer Nationalfonds, the European Community-Research Infrastructure Activity under FP7 programme (Hadron Physics2, grant agreement No. 227431), the UK Science and Technology Facilities Council (STFC 57071/1, 50727/1), the Natural Science and Engineering Research Council (NSERC) in Canada. This material is based upon work supported by the U.S. Department of Energy, Office of Science, Office of Nuclear Physics, under Award Numbers DE-FG02-99-ER41110, DE-FG02-88ER40415, DE-FG02-01-ER41194, and by the National Science Foundation under Grant No. (PHY-1039130 \& IIA-1358175). We thank the undergraduate students of Mount Allison University and The George Washington University for their assistance. M.M. and V.K. thank the Institut für Kernphysik at Mainz where part of this work was performed for hospitality and support.

\section{References}

[1] B. Krusche, Photoproduction of mesons off nuclei, Eur. Phys. J. ST 198 (2011) 199.

[2] Y. Chan et al., Design and performance of a modularized NaI(Tl) detector (the Crystal Ball prototype), IEEE Trans. Nucl. Sci. 25 (1978) 333.

[3] T. D. S. Stanislaus et al., Measurement of neutron detection efficiemcies in NaI using Crystal Ball detector, Nucl. Instrum. Methods A 462 (2001) 463.

[4] J. Alyea et al., Neutrons and the Crystal Ball Experiments, ANL-HEP-TR-97-87.

[5] K.-H. Kaiser et al., The $1.5 \mathrm{GeV}$ harmonic double-sided microtron at Mainz University, Nucl. Instrum. Methods A 593 (2008) 159.

[6] J. C. McGeorge et al., Upgrade of the Glasgow photon tagging spectrometer for Mainz MAMI-C, Eur. Phys. J. A 37 (2008) 129.

[7] A. R. Gabler et al., Response of TAPS to monochromatic photons with energies between 45-MeV and 790-MeV, Nucl. Instrum. Methods A 346 (1994) 168. 
[8] D. Watts, The Crystal Ball and TAPS detectors at the MAMI electron beam facility, Proceedings of the 11-th International Conference on Calorimetry in Particle Physics (Color2004), Perugia, Italy, 2004 (World Scientific, 2005) 560.

[9] G. Audit et al., DAPHNE: A Large acceptance tracking detector for the study of photoreactions at intermediate energies, Nucl. Instrum. Methods A 301 (1991) 473. 